\title{
SOME PHYSIOLOGICAL GENETIC ASPECTS OF MAMMALIAN MELANOGENESIS*
}

\author{
Morris Foster \\ Mammalian Genetics Center, Department of Zoology, The University of \\ Michigan, Ann Arbor, Mich.
}

\section{Introduction: Some Recent Puzzling Findings}

During the past several years we have been studying a number of melanogenic attributes using the skins of house mice afflicted by various types of genetic damage (Foster, 1956, 1959; Foster and Thomson, 1958). Our most recent study (Foster and Thomson, 1961, and unpublished data) involves a closer scrutiny of the effects of allelic interactions at a specific locus, $b$ (brown). Briefly, by means of a combination of manometric and turbidimetric assays we were able to measure the following major sets of attributes: (1) tyrosinase and dopa oxidase activities, (2) initially present natural melanin content, as well as newly formed melanin within incubated skin subsamples, and (3) darkening of incubation media in respirometer vessels due to escape of melanogenic intermediates from incubated skin. These assay procedures are summarized in FIGURE 1.

The results most pertinent to our present discussion are summarized in TABLE 1, but it might be mentioned in passing that we were able to recognize the characteristic numerical profiles of all 10 homozygous and heterozygous genotypes involving the 4 alleles, "light" $\left(B^{L T}\right)$, black $(B)$, cordovan $\left(b^{c}\right)$, and brown $(b)$ - a kind of numerical "taxonomy under glass" for recognition of genotypes.

Focusing attention on the comparisons of black $(B B)$ and brown $(b b)$ homozygotes, as well as the black heterozygote $(B b)$, we find that, depending upon the sets of attributes used for genotypic comparisons, we could conclude that we are dealing with (1) genetic damage when allele $b$ is present, (2) heterotic interaction or enzymic "hybrid vigor" (luxuriance), or (3) the superiority of $b$ over $B$, at least when the skin is incubated with exogenous substrates. These separate and partly incompatible conclusions obviously involve different aspects of the whole truth. One approach involves taking the reasonable position that the organism is trying to tell us it is suffering from a genetic damage when it carries a single or double dose of the brown allele $(b)$. This starting point is supported by the superiority of the black $(B B$ or $B b)$ over brown $(b b)$ skins with respect to the first set of pigmentary attributes listed in TABLE 1 . Thus homozygous $(B B)$ black skin has a higher natural melanin content than brown $(b b)$ skin; it produces more melanin than does brown skin when left to its own endogenous resources (incubation in buffer); moreover, under conditions of substrate saturation (incubation with dopa) when brown skin seems superior to black, the total tissue-bound melanin, both initially present and newly synthesized, nevertheless is found to be greater in black skin. Thus the certain

* The work described in this paper was supported in part by Research Grant C-4305 from the National Cancer Institute, Public Health Service, Bethesda, Md., and in part by Research Grant G-6480 from the National Science Foundation, Washington, D.C. 
genetic damage afflicting the brown skin could involve both (1) some form of endogenous substrate limitation and, almost certainly, (2) a deficiency in the number or effectiveness of the melanin polymer binding sites of the protein granule matrix.

\section{Discussion of Results}

Let us tentatively assume that the brown locus specifies the synthesis of a matrix protein that could be concerned with (1) binding of tyrosinase to neighboring protein molecules in the granule matrix, or (2) provision of conjugation

\section{FROM PROTOPLASM TO NUMBERS}

YOUNG MOUSE

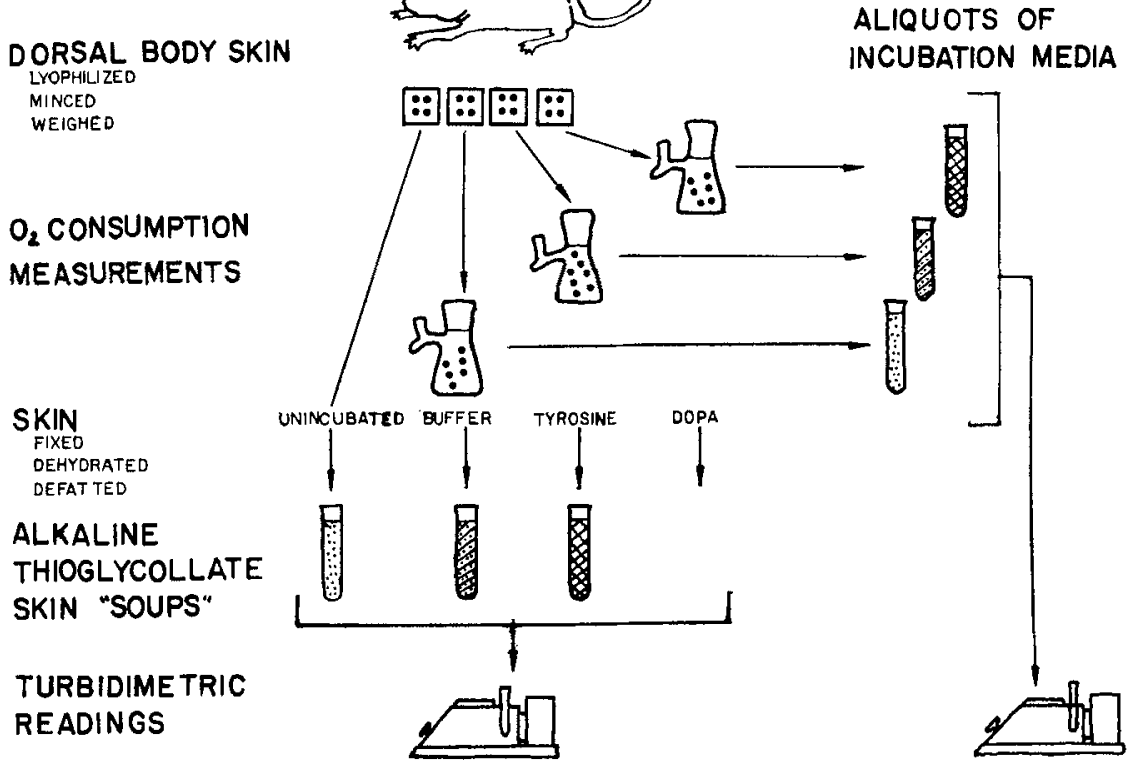

FIGURE 1.

sites for melanin polymers, or (3) the binding of matrix proteins to one another. Moreover, we might visualize a normal melanin granule as a tightly bound complex comprising tyrosinase, as well as other structural and functional proteins. Then a mutation at the brown locus could result in the production of a modified protein that might then secondarily cause an alteration of one or more of the following: (1) location, specific activity, or stability of tyrosinase; (2) number or binding effectiveness of melanin polymer conjugation sites; or (3) the structural integrity of the protein matrix, and hence of the pigment granule.

We have already demonstrated both increased and decreased levels of assayed enzyme activity toward exogenous substrate in the skins containing different mutant alleles; i.e., brown (bb) skin shows increased enzyme activity, while the "light" homozygote $\left(B^{L T} B^{L T}\right)$ shows reduced activity, when both of 
these genotypes are compared with black skin. Moreover, it has been possible to show that these mutants are defective in their melanin-binding capacities under conditions of substrate saturation, i.e., when the dopa incubation media for all genotypes show substantial darkening (intermediates leaking out of the tissue). Thus our observations are consistent with our visualization of the normal melanin granule and of the assumed role of the brown locus.

It still remains for us to try to explain the enzymic "hybrid vigor" (luxuriance) of the black $(B b)$ heterozygote. I can suggest at this point only that the melanin granule matrix of this genotype could contain 2 varieties of the same parent protein molecule, 1 normal and 1 abnormal (altered amino acid sequence), thereby leading to a modified molecular architectural arrangement that secondarily increases the specific activity of tyrosinase. Nevertheless, the end result is that the heterozygote produces less natural pigment than does the black homozygote $(B B)$, perhaps because of the partially reduced number

TABLE 1

SUMmaRized MELANOGENIC INEQUALITIES

\begin{tabular}{|c|c|c|c|}
\hline Attribute & & Genotype ranking & Conclusion \\
\hline $\left.\begin{array}{l}\text { Natural melanin content } \\
\text { Total tissue-bound melanin } \\
\text { Skin darkening in buffer control } \\
\text { medium }\end{array}\right\}$ & (1) & $\mathrm{BB} \geq \mathrm{Bb}>\mathrm{bb}$ & $\begin{array}{l}\text { Genetic damage in single } \\
\text { or double dose of } b\end{array}$ \\
\hline $\left.\begin{array}{l}\text { Tyrosinase, dopa Oxidase } \\
\text { Darkening of tyrosine and dopa in- } \\
\text { cubation media }\end{array}\right\}$ & (2) & $\mathrm{Bb} \geq \mathrm{bb}>\mathrm{BB}$ & "Hybrid vigor" \\
\hline $\left.\begin{array}{l}\text { Skin darkening in tyrosine and } \\
\text { dopa (in excess of control incu- } \\
\text { bated in buffer) }\end{array}\right\}$ & (3) & $\mathrm{bb} \geq \mathrm{Bb}>\mathrm{BB}$ & $\begin{array}{l}b \text { superior to } B \text { in exoge- } \\
\text { nous substrate }\end{array}$ \\
\hline
\end{tabular}

or effectiveness of melanin-binding sites in the "hybrid" granule protein matrix.

\section{General Discussion}

Introductory remarks. In our previous studies of melanogenesis we had often noted no simple correspondence between natural melanin content and other assayed melanogenic attributes. Moreover, the results previously obtained by us seemed amenable to reconciliation by ad hoc invocations of inhibitors, substrate restrictions, or reduced efficiency of terminal melanogenic phases. Such possibilities are still useful, but only partly so, in explaining our current apparently conflicting results.

A number of recently reported facts and ideas provide a basis for developing a broader perspective for interpreting observations concerning melanogenesis. Briefly, the considerations listed below range from postulated relations between genes and proteins to the possible functional consequences of altered macromolecular architecture. The resulting viewpoint is not intended to account for every case of mutational damage. It does, however, provide a basis 
for accepting those genetic situations involving both diminished natural pigmentation and increased tyrosinase activity (assayed under conditions convenient for the observer).

Genetic considerations. A single gene seems to be concerned with specifying the amino acid sequence of a specific polypeptide. At the most only a very few genes contribute to the formation of the primary structure (amino acid sequence) of some specific protein (see review by Yanofsky and St. Lawrence, 1960). Thus a key agent in mammalian melanogenesis, the tyrosinase molecule, is synthesized either under the aegis of a single gene (perhaps the gene for albinism) or of only a very few genes. In Neurospora, for example, only a single gene, $\mathrm{T}$, has so far been implicated in determining 4 structural variants of the tyrosinase molecule (Horowitz et al., 1961). Many other genes affecting melanogenesis in laboratory mammals must then exert their effects by way of other polypeptides or proteins that in various ways regulate the microenvironments in which tyrosinase is synthesized, functions, or is rendered temporarily or permanently inactive.

At the level of the cell, transplantation studies (Silvers, 1961) have shown that at least in the house mouse a number of coat color genes apparently act autonomously within the melanocyte, i.e., the $c$ (albinism), $b$ (brown), $p$ (pink-eyed dilution), $d$ (maltese dilution), and $\ln$ (leaden) loci. At least one other (agouti) locus, however, acts indirectly by controlling the melanocyte tissue environment (see also Cleffmann, 1953, 1954, 1961).

Chemical and cytological considerations. At macromolecular levels one deals with surface-spread protein monolayers, with enzyme-substrate complexes, and with tightly knit architectural relations between a specific enzyme and its large molecular neighbors. Under such conditions of macromolecular architecture, the modified physical state of the enzyme molecule (when compared with the soluble, coiled, "native" state) can be reflected in altered specific activity, stability, or even the degree of intracellular localization (Hayashi, 1952, 1953; Hayashi and Edison, 1950; Kaplan 1952a,b).

It is thus possible to enlarge the repertoire of an enzyme molecule's behavior by virtue of this enzyme's regulation by its macromolecular microenvironment. Such functional alterations could of course be independent of changes in the enzyme's primary amino acid sequence. Thus a number of intracellular organelles (i.e., chromosomes, ribonucleoprotein granules, mitochondria, and, of course, melanin granules) provide a basis for regulatory interactions between some key substances (such as nucleic acids or enzyme proteins) and the proteins with which these key substances are conjugated (see also Nanney, 1960, and Markert, 1958).

The possibility of such regulatory interactions is intriguing because immediate neighborhood proteins could provide the vehicles, and specific organelles could provide the foci, for numerous patterns of genic interactions. Indeed it seems conceivable that a critical stage in biochemical evolution or in cellular differentiation might in some cases depend, respectively, upon the origin or "activation" ("turning on") of a single gene controlling the synthesis of some specific "architectural" protein. Such an "architectural" protein, by virtue of its binding sites, could convert a somewhat disordered array of different 
large key molecules into the highly ordered arrangement characteristic of a normal intracellular organelle (see also von Wettstein, 1959).

Thus evolutionary or epigenetic complexity could depend as much upon the variety of stablized patterns of genic, and consequently of protein, interactions as upon the absolute number of different genes and of corresponding proteins.

At the organelle level of melanin granule construction, electron microscope studies (Birbeck and Barnicot, 1959; Seiji et al., 1961; Birbeck, 1961; Wellings and Siegel, 1961; Moyer, 1961) are consistent with the following presumed sequence of events: (1) the initial synthesis and assembling of granule matrix proteins, (2) the localized attachment of the already synthesized tyrosinase molecule to particular binding sites on the internal membranes of the protein matrix or "premelanin" granule, (3) the spatially organized enzymic conversion of amino acid precursor(s) to melanin polymers, and (4) the conjugation of melanin polymers with specific binding sites of the protein matrix.

The melanin granule and melanogenesis. In view of the foregoing, we might visualize a normal melanin granule consisting of a number of different proteins, including tyrosinase. A number of genes could act within the melanocyte by controlling the synthesis of different granule matrix proteins. These proteins could assume different roles: (1) to localize tyrosinase and melanin polymers, (2) to bind matrix proteins together, (3) to provide melanogenic substrates. A gene mutation resulting in a modified matrix protein could then simultaneously produce a number of secondary consequences: (1) altered tyrosinase activity, (2) changed availability of endogenous substrates, (3) modified degree of melanization, (4) altered melanin granule structure.

It is thus possible now to harmonize our apparently conflicting sets of observations obtained from the same tissue.

\section{Concluding Remarks}

Our visualization of architectural and functional relations within the normal melanin granule, and the possible consequences arising from genetic damage, have at least helped us transfer a group of observations from a category of interesting curiosities into a framework sufficiently broad to embrace a number of unexpected correlated findings. Such a framework seems almost too broadtoo many ad hoc explanations seem possible, and too few specific predictions.

It seems conceivable, however, that one assumption might lend itself to testing in the foreseeable future, namely, that of the heterogeneous protein composition of the granule matrix. Perhaps, too, the combined powerful approaches of electron microscopy and of physical biochemistry, when applied to detailed comparisons between normal and genetically controlled abnormal situations, might lead to a sharper viewpoint with greater predictive value.

\section{Acknowledgment}

On the occasion of L. S. Dunn's retirement from the Department of Zoology at Columbia University, I express my great indebtedness to him for his continuing stimulation and encouragement, and for his exemplary embodiment of the finest attributes of the teaching scholar.

\section{References}

BIRBECK, M. S. C. 1963. Electron microscopy of melanocytes: The Fine Structure of Hair-Bulb Premelanosomes. Ann. N.Y. Acad. Sci. 100: 540-547. 
BirbeCK, M. S. C. \& N. A. Barnicot. 1959. Electron microscope studies on pigment formation in human hair follicles. In Pigment Cell Biology. : 549-561. Myron Gordon, Ed. Academic Press. New York, N.Y.

Clefrmann, G. 1953. Untersuchungen über die Fellzeichnung des Wildkaninchens. Ein Beitrag zur Wirkungsweise des Agutifaktors. Z. Ind. Abst. Vererb. 85: 137-162.

Clefrmann, G. 1954. Über die Beeinflussung der Wildfärbung in vitro. Z. Naturforsch. 9b: 701-704.

Cleffrmann, G. 1963. Ann. N.Y. Acad. Sci. 100: 749-761.

Foster, M. 1956. Inherited variations in the pigmentary metabolism of mouse skin. Genetics. 41: 643. (Abstract.)

Foster, M. 1959. Physiological studies of melanogenesis. In Pigment Cell Biology. : 301314. Myron Gordon, Ed. Academic Press. New York, N.Y.

Foster, M. \& L. Thomson. 1958. The effects of substitution at the leaden (ln) locus on melanogenic attributes of the mouse. Proc. X Int. Congr. Genetics. II: 84 . (Abstract.)

Foster, M. \& L. Thomson. 1961. Allelic relations at the brown locus of the house mouse. Genetics. 46: 865. (Abstract.)

Hayashi, T. 1952. Contractile properties of compressed monolayers of actomyosin. J. Gen. Physiol, 36: 139-152.

Hayashi, T. 1953. Surface-spread protein as a basis for cell structure and cell movement. Am. Naturalist. 87: 209-227.

Hayashi, T. \& G. R. EDISON. 1950. Enzyme-substrate stabilization with surface-denatured pepsin-albumin. J. Coll. Sci. 5: 437-447.

Horowitz, N. H., M. Fling, H. Macleod \& N. Sueoka. 1961. A genetic study of two new structural forms of tyrosinase in Neurospora. Genetics. 46: 1015-1024.

KAPLAN, J. G. 1952a. The biological activity and physical state of intracellular catalase. Physiol. Zool. 25: 123-131.

KAPLAN, J. G. 1952b. The activity of catalase unfolded at the air-water interface. J. Coll. Sci. 7: 382-395.

MARKERT, C. L. 1958. Chemical concepts of cellular differentiation. In The Chemical Basis of Development. : 3-16. W. D. McElroy and H. B. Glass, Eds. Johns Hopkins Press. Baltimore, Md.

Mover, F. H. 1961. The fine structure of melanosomes in normal and pathological tissues. Ann. N.Y. Acad. Sci.

NANNEY, D. L. 1960. Microbiology, developmental genetics and evolution. Am. Naturalist. 94: $167-180$.

Seiji, M., T. B. Fitzpatrick \& K. Shimao. 1961. The melanosome, a distinct entity. Ann. N.Y. Acad. Sci.

SILVERS, W. K. 1961. Genes and the pigment cells of mammals. Science. 134: 368-373.

voN WeTtstein, D. 1959. Developmental changes in chloroplasts and their genetic control. In Developmental Cytology. 16th Symp. Soc. Study of Development and Growth. : 123-160. Dorothea Rudnick, Ed.

WELlings, S. R. \& B. V. SIEgeL. 1963. Electron microscopic studies on the subcellular origin and ultrastructure of melanin granules in mammalian melanomas. Ann. N.Y. Acad. Sci. 100: 548-568.

Yanofsky, C. \& P. St. Lawrence. 1960. Gene action. Ann. Rev. Microbiol. 14:311-340.

\section{Discussion of the Paper}

F. H. Moyer, JR. (Johns Hopkins University Medical School, Baltimore, Md.): Based on electron microscope pictures that we have obtained, we think that the protein matrix of the melanin granule is composed of at least three subunits, since in the albino animal we find a well-organized but unmelanized matrix; whereas in the pink-eyed animal we see melanization but a disorganized matrix. In black and brown animals, both organization and melanization occur, but the melanin deposited on the matrix is different.

This clearly implies to us that there are three structural subunits under different genetic control. They are probably controlled by distinct genetic loci on three separate chromosomes.

M. Foster (University of Michigan, Ann Arbor, Mich.): According to Dr. Silver, we can now also entertain the notion that there are at least five genecontrolled proteins in a matrix, as shown by his grafting experiments. 